\title{
Energy Balancing of the Modular Multilevel Matrix Converter based on a New Transformed Arm Power Analysis
}

\author{
Felix Kammerer, Mario Gommeringer, Johannes Kolb², Michael Braun \\ Karlsruhe Institute of Technology (KIT) \\ Elektrotechnisches Institut (ETI) - Electrical Drives and Power Electronics \\ Kaiserstr. 12, 76131 Karlsruhe, Germany \\ felix.kammerer@kit.edu,www.eti.kit.edu \\ ${ }^{2}$ Schaeffler Technologies GmbH \& Co. KG - SHARE at KIT
}

\section{Acknowledgments}

The authors would like to thank the DFG (German Research Foundation) which supports this research project.

\section{Keywords}

$<<$ AC/AC converter $>>,<<$ Multilevel converters $>>,<<$ Variable speed drive $>>$

\begin{abstract}
This paper presents a transformed arm power analysis of the Modular Multilevel Matrix Converter (M3C). It enables the energy balancing in the whole frequency range for high power variable-speed drive applications. Four balancing directions are identified for the active power exchange between the converter arms with minimal internal currents. At critical operating points a zero sequence voltage is used. Additionally, the reactive power components can be used to perform a real time calculation of the energy pulsation in all four balancing directions to improve the control performance. A low voltage prototype with 5 cells in each of the nine arms has been realized to verify the theoretical analysis.
\end{abstract}

\section{Introduction}

The investigated Modular Multilevel Matrix Converter (M3C) is shown in Fig. 1. It performs a direct three-phase $\mathrm{AC}$ to $\mathrm{AC}$ voltage conversion. For example it is suitable for high power variable-speed drive applications with low nominal frequencies. The M3C can be used to replace line commuted cycloconverters in the future due to its modular and scalable structure as well as its high voltage quality on the input and output side which avoids filters. For applications with nominal frequencies greater than grid frequency it is also a advantageous choice in the medium voltage range due to the fact that the effective switching frequency for the semiconductors in the $\mathrm{M} 3 \mathrm{C}$ is much lower than the resulting switching frequency for the input and output side.

Basic research of the $\mathrm{M} 3 \mathrm{C}$ without arm inductors has been presented in [1,2] together with corresponding space vector control schemes. There, the converter arms work as controllable voltage sources which implies that exactly five of the nine converter arms must conduct arm currents at the same time to avoid short circuits at the input or the output side. The results obtained by these methods are difficult to implement in a M3C with a higher number $N$ of cells per arm due to the very high number of switching states, which must be computed and selected in real time. An additional problem is the current commutation between converter arms which causes high-current peaks, even in small prototypes with $N=1$ cell per arm [2]. These current peaks must be limited by additional arm inductors.

Further improvements, using nine arm inductors and corresponding open-loop or cascaded feedback control methods are presented in $[3,4,5,6]$. Here the M3C converter arms work as controllable current sources with continuously flowing arm currents in all nine converter arms. This completely avoids short circuits between the converter arms.

The M3C with three-phase coupled z-winding arm inductors $L$ (Fig. 1) was first presented in [7]. A cascaded vector control scheme was introduced to balance the nine arm energies which are represented 


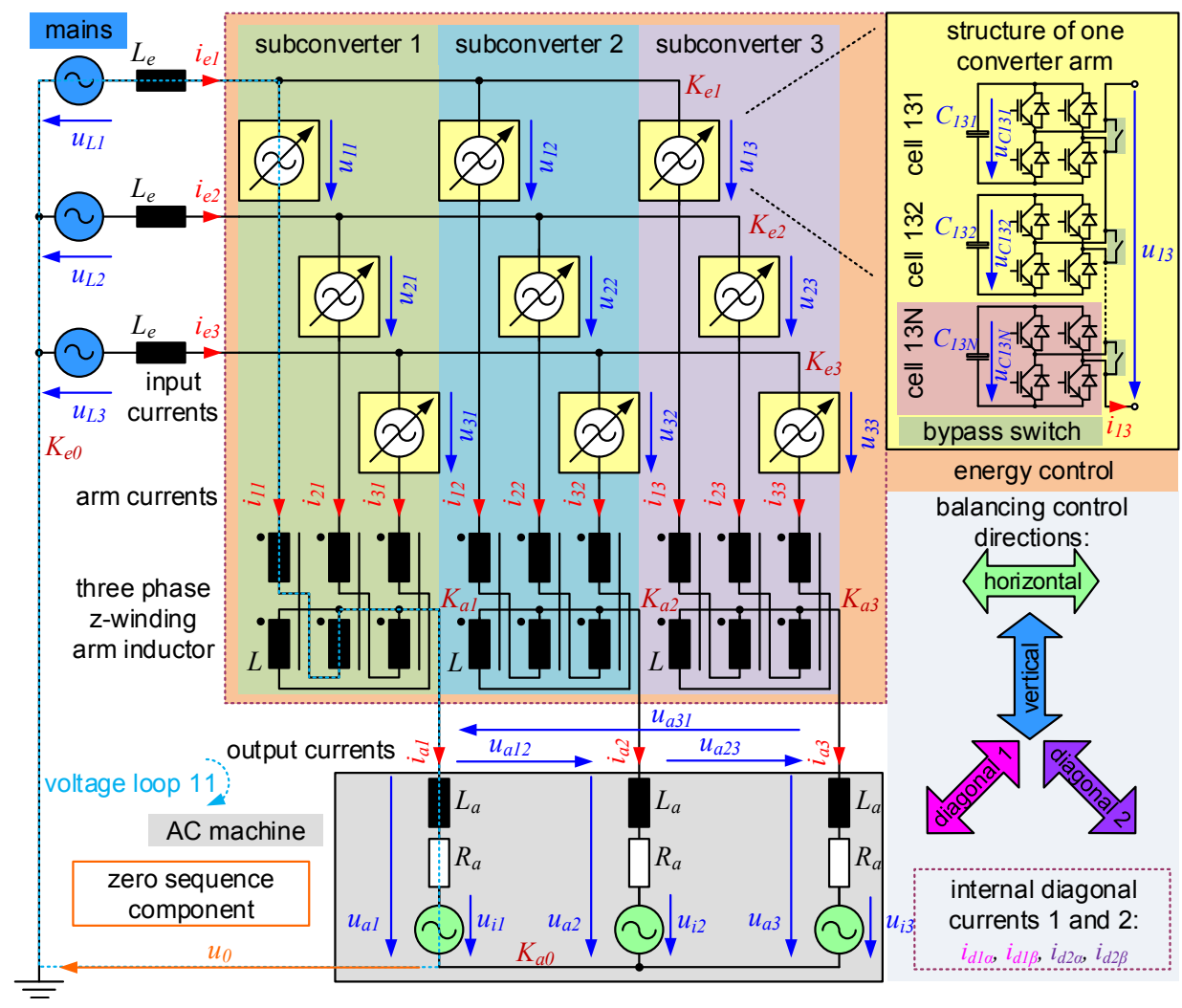

Figure 1: Modular Multilevel Matrix Converter (M3C) with coupled three-phase z-winding arm inductors L. The $\mathrm{M} 3 \mathrm{C}$ converter arms consist of $\mathrm{N}$ series connected $\mathrm{H}$-bridges with a DC-capacitor $C_{x y z}$.

by the sum of the capacitor voltages in the nine converter arms. A modulator selects the cells being switched on to apply the arm voltage reference values. This is done based on a sorting algorithm which balances the cell capacitor voltages inside of each converter arm. The cascaded vector control scheme from [7] was extended in [8] with additional transformation steps to separate input, output and internal currents. [9] gives a detailed description of the $\mathrm{z}$-winding arm inductor $L$ which allows considerable savings of core and winding material compared to nine individual arm inductors. This is possible due to the fact that the output currents $i_{a 1}, i_{a 2}, i_{a 3}$ do not produce any flux inside the inductor core. As a result, the $\mathrm{M} 3 \mathrm{C}$ converter arms work as controllable voltage sources to feed the AC machine and as controllable current sources for the input and the internal currents. The advantages of using the M3C to feed the rotor winding of a doubly fed induction generator (DFIG) is shown in [10]. This variable speed generator system consisting of the M3C and the DFIG improves the dynamic behavior and can increase the part load efficiency of power plants compared to grid-connected synchronous generators.

[11] shows an alternative vector control scheme and a method to reduce the energy pulsation at very low output frequencies using a phase modulation of the reference values for the internal currents instead of a amplitude modulation [6]. [12] shows a method to reduce the energy pulsation in the case that the input and the output frequency are similar. [11] calculates transformed power components which can be used to design the balancing control of the M3C. Experimental results with $N=4$ cells per arm showing steady-state operation of the $\mathrm{M} 3 \mathrm{C}$ are given in $[11,12,13]$.

In this paper, a transformed arm power analysis of the $\mathrm{M} 3 \mathrm{C}$ based on the transformed vector control scheme in [8] and the circuit and power analysis given in [7] and [9] will be introduced, having the following advantages:

- The transformation allows an easier identification of the relevant current and voltage components which generate active power components for the energy balancing in four different directions (Fig.1). Unnecessary current stress on the converter arms can be avoided by using only the minimally necessary internal currents.

- Especially, if the absolute input and output frequencies are equal, specific active power components must be compensated. [12] presents a method using internal currents without any zero sequence voltage, which allows the operation under certain constraints. Here, the method is extended by using the zero sequence voltage as an additional degree of freedom, allowing an expanded operating range and a stationary operation at the same absolute frequency.

- The reactive power components are separated into their different frequencies. This allows an easier online computation of the energy pulsation. The dynamic behavior is improved by avoiding filters in the feedback paths of the balancing control.

- The cascaded control system can be directly derived based on the transformed power analysis. 


\section{Structure and fundamentals of the M3C}

The overall system including the three-phase AC grid, the Modular Multilevel Matrix Converter (M3C) and the three-phase machine is shown in Fig. 1. The M3C consists of three subconverters, each of them connects the three input phases via three converter arms and the three phase z-winding arm inductor $L$ to one output phase. As can be seen from Fig. 1, the converter arms are modeled as controllable voltage sources and consist of $N$ series connected cells. They are realized as H-bridges with a capacitor $C_{x y z}(x=$ input phase number, $y=$ output phase number, $x, y \in\{1,2,3\}, z=$ cell number, $z \in\{\mathbb{N} \mid 1 \leq z \leq N\}$ ). By switching the cells, each arm is able to generate an adjustable arm voltage $u_{x y}$ with $2 N+1$ voltage steps. The specific voltage range of the arms involving at least one cell with pulse width modulation (PWM) [7] is:

$$
-u_{C x y}=-\sum_{z=1}^{N} u_{C x y z} \leq u_{x y} \leq u_{C x y}=\sum_{z=1}^{N} u_{C x y z}
$$

$u_{C x y}$ is the arm capacitor voltage which is equal to the sum of all cell capacitor voltages $u_{C x y z}$ in the arm $x y$. The arm capacitor voltages $u_{C x y}$ can be split into a constant average value $\bar{u}_{C}$ corresponding to the mean capacitor energy $\bar{w}_{C}$ stored in the converter arms and a time-variant part $\tilde{u}_{C x y}$ which depends on the actual arm power $p_{x y}$ :

$$
u_{C x y}=\tilde{u}_{C x y}+\bar{u}_{C} \approx \frac{N}{C_{x y z} \cdot \bar{u}_{C}} \int p_{x y} d t+\bar{u}_{C}
$$

To maintain a constant average arm capacitor voltage $\bar{u}_{C}$, the control system has to ensure that the average arm power $\bar{p}_{x y}$ is zero for all nine converter arms.

The Scalability of the M3C in the voltage and power range is fully provided. With a higher number $N$ of cells per arm, a higher arm voltage $u_{x y}$ and therefore a higher power can be achieved. The operation of the $\mathrm{M} 3 \mathrm{C}$ continues in case of component failures with a reduced output voltage $u_{a y}$ by short-circuiting failed cells with a bypass switch (Fig. 1). Additional redundancy cells can be installed in cases where the output voltage reduction is not permitted after cell failures.

\subsection{Transformed equivalent circuit analysis of the M3C}

In this Section, the $\mathrm{M} 3 \mathrm{C}$ is analyzed to calculate the transformed equivalent circuits for the independent current control of input, output and internal diagonal currents. Additionally, the composition of the arm voltages and currents is calculated for the arm power analysis in Section 2.

The Kirchhoff's voltage law for each of the three subconverters $y \in\{1,2,3\}$ is given by (Fig. 1):

$$
-\left[\begin{array}{l}
u_{L 1} \\
u_{L 2} \\
u_{L 3}
\end{array}\right]+L_{e} \cdot \frac{d}{d t}\left[\begin{array}{l}
i_{e 1} \\
i_{e 2} \\
i_{e 3}
\end{array}\right]+\left[\begin{array}{c}
u_{1 y} \\
u_{2 y} \\
u_{3 y}
\end{array}\right]+L \cdot \frac{d}{d t}\left[\begin{array}{l}
i_{1 y} \\
i_{2 y} \\
i_{3 y}
\end{array}\right]+\left[\begin{array}{c}
u_{a y} \\
u_{a y} \\
u_{a y}
\end{array}\right]+\left[\begin{array}{l}
u_{0} \\
u_{0} \\
u_{0}
\end{array}\right]=0 \quad y \in\{1,2,3\}
$$

The dashed blue line in Fig. 1 is equal to the first row of (3) for subconverter 1 and illustrates the voltage loop 11. The amplitude invariant $\alpha \beta 0$-transformation matrix $C_{\alpha \beta 0}$ (4) allows to calculate orthogonal $\alpha \beta$-components and the independent zero sequence component 0 of three time variant values, e.q. three currents or three voltages.

$$
\left[\begin{array}{l}
i_{\alpha} \\
i_{\beta} \\
i_{0}
\end{array}\right]=C_{\alpha \beta 0} \cdot\left[\begin{array}{l}
i_{1} \\
i_{2} \\
i_{3}
\end{array}\right], \quad\left[\begin{array}{l}
u_{\alpha} \\
u_{\beta} \\
u_{0}
\end{array}\right]=C_{\alpha \beta 0} \cdot\left[\begin{array}{l}
u_{1} \\
u_{2} \\
u_{3}
\end{array}\right], \quad C_{\alpha \beta 0}=\left[\begin{array}{ccc}
\frac{2}{3} & -\frac{1}{3} & -\frac{1}{3} \\
0 & \frac{1}{\sqrt{3}} & -\frac{1}{\sqrt{3}} \\
\frac{1}{3} & \frac{1}{3} & \frac{1}{3}
\end{array}\right]
$$

This fact is used to transform the voltage loops into the $\alpha \beta$-components and the independent zero sequence component 0 from (3) for each of the three subconverters:

$$
-\left[\begin{array}{c}
u_{L \alpha} \\
u_{L \beta} \\
0
\end{array}\right]+L_{e} \cdot \frac{d}{d t}\left[\begin{array}{c}
i_{e \alpha} \\
i_{e \beta} \\
0
\end{array}\right]+\left[\begin{array}{c}
u_{\alpha y} \\
u_{\beta y} \\
u_{0 y}
\end{array}\right]+L \cdot \frac{d}{d t}\left[\begin{array}{c}
i_{\alpha y} \\
i_{\beta y} \\
i_{0 y}=\frac{i_{a y}}{3} \approx 0
\end{array}\right]+\left[\begin{array}{c}
0 \\
0 \\
u_{a y}
\end{array}\right]+\left[\begin{array}{c}
0 \\
0 \\
u_{0}
\end{array}\right]=0 \quad y \in\{1,2,3\}
$$

The third row of (5) contains the zero sequence components consisting of the output voltages $u_{a y}$ and the zero sequence voltage $u_{o}$. The output currents $i_{a y}$ can be calculated by applying Kirchhoff's current law to the output connection points $K_{a y}(y \in\{1,2,3\})$ (Fig. 1):

$$
i_{a y}=i_{1 y}+i_{2 y}+i_{3 y}=3 \cdot i_{0 y} \quad y \in\{1,2,3\}
$$


Here each converter arm delivers the zero sequence current $i_{0 y}$ which is equal to one third of the phase output current $i_{a y}$. By using the coupled three phase z-winding arm inductors $L$ the output currents $i_{a y}$ only produce a small stray flux in the inductor cores, hence the voltages $L \cdot \frac{d}{d t} i_{0 y}=L \cdot \frac{d}{d t} \frac{i_{a y}}{3} \approx 0$ [9].

The second transformation step using the matrix $C_{\alpha \beta 0}(4)$ is applied line by line to (5). The transformation of the three $\alpha y$-components, the three $\beta y$-components and the three $0 y$-components results in:

$$
\begin{aligned}
& L_{e} \cdot \frac{d}{d t}\left[\begin{array}{c}
0 \\
0 \\
i_{e \alpha}
\end{array}\right]+L \cdot \frac{d}{d t}\left[\begin{array}{c}
i_{\alpha \alpha} \\
i_{\alpha \beta} \\
i_{\alpha 0}=\frac{i_{e \alpha}}{3}
\end{array}\right]=\left[\begin{array}{c}
0 \\
0 \\
u_{L \alpha}
\end{array}\right]-\left[\begin{array}{c}
u_{\alpha \alpha} \\
u_{\alpha \beta} \\
u_{\alpha 0}
\end{array}\right] \\
& L_{e} \cdot \frac{d}{d t}\left[\begin{array}{c}
0 \\
0 \\
i_{e \beta}
\end{array}\right]+L \cdot \frac{d}{d t}\left[\begin{array}{c}
i_{\beta \alpha} \\
i_{\beta \beta} \\
i_{\beta 0}=\frac{i_{e \beta}}{3}
\end{array}\right]=\left[\begin{array}{c}
0 \\
0 \\
u_{L \beta}
\end{array}\right]-\left[\begin{array}{c}
u_{\beta \alpha} \\
u_{\beta \beta} \\
u_{\beta 0}
\end{array}\right] \\
& L_{a} \cdot \frac{d}{d t}\left[\begin{array}{c}
i_{a \alpha} \\
i_{a \beta} \\
i_{00}=0
\end{array}\right]+\left[\begin{array}{c}
0 \\
0 \\
u_{0}
\end{array}\right]=-R_{a} \cdot\left[\begin{array}{c}
i_{a \alpha} \\
i_{a \beta} \\
i_{00}=0
\end{array}\right]-\left[\begin{array}{c}
u_{i \alpha} \\
u_{i \beta} \\
0
\end{array}\right]-\left[\begin{array}{c}
u_{0 \alpha} \\
u_{0 \beta} \\
u_{00}
\end{array}\right]
\end{aligned}
$$

The input currents $i_{e \alpha}$ and $i_{e \beta}$ can be calculated by applying Kirchhoff's current law to the input connection points $K_{e x}(x \in\{1,2,3\})$. After the two transformation steps they can be expressed by their $\alpha \beta$-components:

$$
i_{e \alpha}=i_{\alpha 1}+i_{\alpha 2}+i_{\alpha 3}=3 \cdot i_{\alpha 0} \quad i_{e \beta}=i_{\beta 1}+i_{\beta 2}+i_{\beta 3}=3 \cdot i_{\beta 0}
$$

Each converter arm sinks the zero sequence currents $i_{\alpha 0}, i_{\beta 0}$ which are equal to one third of the input currents $i_{e \alpha}, i_{e \beta}$. The third line of (7) and (8) leads to the corresponding equivalent circuits for the input currents $i_{e \alpha}, i_{e \beta}$ (Fig. 2a)). They are orientated vertical in the M3C (Fig. 1). The first and second line of (9) leads to the equivalent circuits for the horizontal orientated (Fig. 1) output currents $i_{a \alpha}, i_{a \beta}$ (Fig. 2b)). The third line contains the zero sequence circuit (Fig. 2c)). The points $K_{e 0}$ and $K_{a 0}$ are not connected, hence the zero sequence current is $i_{00}=0$ and the circuit remains open. The first and second line of
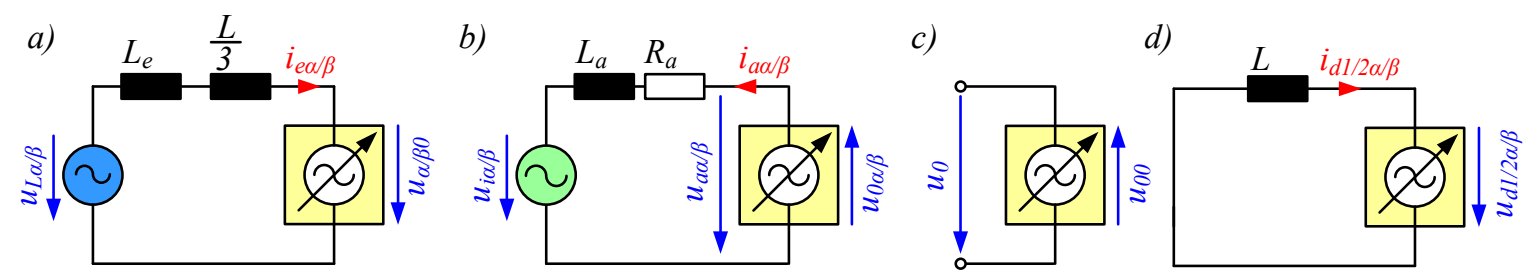

Figure 2: a) Equivalent circuit for the input currents $i_{e \alpha / \beta}$, b) Equivalent circuit for the output currents $i_{a \alpha / \beta} \mathrm{c}$ ) Equivalent circuit for the zero sequence voltage $u_{0}$ d) Equivalent circuit for the internal diagonal currents $i_{d 1 / 2 \alpha / \beta}$

(7) and (8) represent the equivalent circuits for the internal currents $i_{\alpha \alpha}, i_{\alpha \beta}, i_{\beta \alpha}$ and $i_{\beta \beta}$. These currents sum up to zero at the points $K_{e x}$ and $K_{a y}$ and do not have any influence to the input or the output side of the M3C. They are used for energy balancing or reducing the energy pulsation in the converter arms $[8,11]$. Otherwise, they are controlled to zero. To obtain a geometrical orientation of the four currents, the diagonal transformation matrix $C_{D}$ is introduced:

$$
\left[\begin{array}{l}
i_{d 1 \alpha} \\
i_{d 1 \beta} \\
i_{d 2 \alpha} \\
i_{d 2 \beta}
\end{array}\right]=C_{D} \cdot\left[\begin{array}{l}
i_{\alpha \alpha} \\
i_{\alpha \beta} \\
i_{\beta \alpha} \\
i_{\beta \beta}
\end{array}\right], \quad\left[\begin{array}{l}
u_{d 1 \alpha} \\
u_{d 1 \beta} \\
u_{d 2 \alpha} \\
u_{d 2 \beta}
\end{array}\right]=C_{D} \cdot\left[\begin{array}{l}
u_{\alpha \alpha} \\
u_{\alpha \beta} \\
u_{\beta \alpha} \\
u_{\beta \beta}
\end{array}\right], \quad C_{D}=\left[\begin{array}{cccc}
+\frac{1}{2} & 0 & 0 & +\frac{1}{2} \\
0 & +\frac{1}{2} & -\frac{1}{2} & 0 \\
+\frac{1}{2} & 0 & 0 & -\frac{1}{2} \\
0 & +\frac{1}{2} & +\frac{1}{2} & 0
\end{array}\right]
$$

Applying (11) to the first and second line of (7) and (8) results in two orthogonal systems expressed by their $\alpha \beta$-components:

$$
L \cdot \frac{d}{d t}\left[\begin{array}{l}
i_{d 1 \alpha} \\
i_{d 1 \beta} \\
i_{d 2 \alpha} \\
i_{d 2 \beta}
\end{array}\right]=-\left[\begin{array}{l}
u_{d 1 \alpha} \\
u_{d 1 \beta} \\
u_{d 2 \alpha} \\
u_{d 2 \beta}
\end{array}\right]
$$


The corresponding equivalent circuits are shown in Fig. 2d) and they are orientated in diagonal 1 and diagonal 2 direction, see Fig. 1.

As a result, the transformation performs a calculation of nine equivalent circuits (Fig.2) with their corresponding voltage and current components. This allows the independent design and tuning of the current controllers for the input currents $i_{e \alpha / \beta}$, the output currents $i_{a \alpha / \beta}$ and the diagonal currents $i_{d 1 / 2 \alpha / \beta}$ [8].

The transformed arm voltage reference values, calculated by the eight current controllers, and the zero sequence voltage must be transformed back stepwise using the inverse matrices $C_{D}^{-1}$ and $C_{\alpha \beta 0}^{-1}$ to obtain the arm voltages $u_{x y}$ which must be applied to the nine converter arms:

$$
\begin{aligned}
& {\left[\begin{array}{l}
i_{\alpha \alpha} \\
i_{\alpha \beta} \\
i_{\beta \alpha} \\
i_{\beta \beta}
\end{array}\right]=C_{D}^{-1} \cdot\left[\begin{array}{l}
i_{d 1 \alpha} \\
i_{d 1 \beta} \\
i_{d 2 \alpha} \\
i_{d 2 \beta}
\end{array}\right], \quad\left[\begin{array}{l}
u_{\alpha \alpha} \\
u_{\alpha \beta} \\
u_{\beta \alpha} \\
u_{\beta \beta}
\end{array}\right]=C_{D}^{-1} \cdot\left[\begin{array}{l}
u_{d 1 \alpha} \\
u_{d 1 \beta} \\
u_{d 2 \alpha} \\
u_{d 2 \beta}
\end{array}\right], \quad C_{D}^{-1}=\left[\begin{array}{cccc}
+1 & 0 & +1 & 0 \\
0 & +1 & 0 & +1 \\
0 & -1 & 0 & +1 \\
+1 & 0 & -1 & 0
\end{array}\right]} \\
& {\left[\begin{array}{l}
i_{1} \\
i_{2} \\
i_{3}
\end{array}\right]=C_{\alpha \beta 0}^{-1} \cdot\left[\begin{array}{l}
i_{\alpha} \\
i_{\beta} \\
i_{0}
\end{array}\right], \quad\left[\begin{array}{l}
u_{1} \\
u_{2} \\
u_{3}
\end{array}\right]=C_{\alpha \beta 0}^{-1} \cdot\left[\begin{array}{l}
u_{\alpha} \\
u_{\beta} \\
u_{0}
\end{array}\right], \quad C_{\alpha \beta 0}^{-1}=\left[\begin{array}{ccc}
+1 & 0 & +1 \\
-\frac{1}{2} & +\frac{\sqrt{3}}{2} & +1 \\
-\frac{1}{2} & -\frac{\sqrt{3}}{2} & +1
\end{array}\right]}
\end{aligned}
$$

For example, the resulting arm voltage $u_{11}$ consists of the following transformed voltage components:

$$
u_{11}=u_{\alpha 0}+u_{d 1 \alpha}+u_{d 2 \alpha}+u_{0 \alpha}+u_{00}
$$

Due to their low values, the voltages applied to the input inductor $L_{e}$ and the arm inductors $L$ are neglected for the following analysis. Hence the arm voltage $u_{11}$ can be approximated to:

$$
u_{11} \approx u_{L \alpha}-u_{a \alpha}-u_{0}
$$

The resulting arm current $i_{11}$ consists of the following transformed current components:

$$
i_{11}=\frac{i_{e \alpha}}{3}+\frac{i_{a \alpha}}{3}+i_{d 1 \alpha}+i_{d 2 \alpha}
$$

As result of the transformed circuit analysis it can be seen that the $\mathrm{M} 3 \mathrm{C}$ arm currents $i_{x y}$ and arm voltages $u_{x y}$ can be described as four superposed orthogonal systems expressed by their respective $\alpha \beta$-components and one zero sequence component. Each AC system is orientated to one of the four directions shown in Fig. 1 and marked with the respective color. Note that some of the systems can be zero, e.q. the zero sequence current $i_{00}$.

\section{Transformed arm power analysis}

In this Section, each arm power $p_{x y}$ is transformed and analyzed. This allows to identify the transformed arm current and voltage components to generate active power components for the energy and balancing control. Additionally, the reactive power components can be identified for the dimensioning of the cell capacitors $C_{x y z}$. The reactive power components are also needed for the real time calculation of the voltage pulsation $\tilde{u}_{C x y}$. This improves the balancing control performance by avoiding low-pass filters in the feedback paths of the balancing control.

The instantaneous arm power $p_{x y}$ of the $\mathrm{M} 3 \mathrm{C}$ can be calculated with the arm voltage $u_{x y}$ and the arm current $i_{x y}$ :

$$
p_{x y}=u_{x y} \cdot i_{x y}
$$

For example, the calculation of the arm power $p_{11}$ using (16) and (17) results in:

$$
p_{11}=u_{11} \cdot i_{11} \approx\left(u_{L \alpha}-u_{a \alpha}-u_{0}\right) \cdot\left(\frac{i_{e \alpha}}{3}+\frac{i_{a \alpha}}{3}+i_{d 1 \alpha}+i_{d 2 \alpha}\right)
$$

Equation (19) shows that the independent superposed voltage and current systems are coupled via the resulting arm power. Calculating the other converter arms shows that the resulting arm power equations are complex to analyze systematically. To simplify the analysis, the arm power is transformed in the 
same way as the currents and voltages. This leads to four independent orthogonal systems and a zero sequence component. Here the transformation matrix $C_{\alpha \beta 0}(4)$ is used to transform the arm power $p_{x y}$ of the three subconverters:

$$
\left[\begin{array}{c}
p_{\alpha 1} \\
p_{\beta 1} \\
p_{01}
\end{array}\right]=C_{\alpha \beta 0} \cdot\left[\begin{array}{l}
p_{11} \\
p_{21} \\
p_{31}
\end{array}\right], \quad\left[\begin{array}{c}
p_{\alpha 2} \\
p_{\beta 2} \\
p_{02}
\end{array}\right]=C_{\alpha \beta 0} \cdot\left[\begin{array}{l}
p_{12} \\
p_{22} \\
p_{32}
\end{array}\right], \quad\left[\begin{array}{c}
p_{\alpha 3} \\
p_{\beta 3} \\
p_{03}
\end{array}\right]=C_{\alpha \beta 0} \cdot\left[\begin{array}{l}
p_{13} \\
p_{23} \\
p_{33}
\end{array}\right]
$$

The second transformation step transforms (20) line by line using $C_{\alpha \beta 0}$ :

$$
\left[\begin{array}{c}
p_{\alpha \alpha} \\
p_{\alpha \beta} \\
p_{\alpha 0}
\end{array}\right]=C_{\alpha \beta 0} \cdot\left[\begin{array}{c}
p_{\alpha 1} \\
p_{\alpha 2} \\
p_{\alpha 3}
\end{array}\right], \quad\left[\begin{array}{c}
p_{\beta \alpha} \\
p_{\beta \beta} \\
p_{\beta 0}
\end{array}\right]=C_{\alpha \beta 0} \cdot\left[\begin{array}{c}
p_{\beta 1} \\
p_{\beta 2} \\
p_{\beta 3}
\end{array}\right], \quad\left[\begin{array}{c}
p_{0 \alpha} \\
p_{0 \beta} \\
p_{00}
\end{array}\right]=C_{\alpha \beta 0} \cdot\left[\begin{array}{l}
p_{01} \\
p_{02} \\
p_{03}
\end{array}\right]
$$

In the third step the diagonal transformation matrix $C_{D}(11)$ is used:

$$
\left[\begin{array}{l}
p_{d 1 \alpha} \\
p_{d 1 \beta} \\
p_{d 2 \alpha} \\
p_{d 2 \beta}
\end{array}\right]=C_{D} \cdot\left[\begin{array}{l}
p_{\alpha \alpha} \\
p_{\alpha \beta} \\
p_{\beta \alpha} \\
p_{\beta \beta}
\end{array}\right]
$$

The resulting transformed arm power components are shown in Table I. They correspond to the four balancing directions of Fig. 1 and influence the nine arm capacitor voltages $u_{C x y}$ according to (2). They can be expressed by nine transformed values after applying the transformation steps from (20) - (22) to the nine arm capacitor voltages $u_{C x y}$.

Table I: Transformed arm capacitor voltages influenced by the transformed arm power components

\begin{tabular}{|c|c|c|c|c|}
\hline \multicolumn{2}{|c|}{ transformed arm power } & $(2)$ & \multicolumn{2}{c|}{ transformed arm capacitor voltage } \\
\hline$p_{00}$ & average arm power & $\Rightarrow$ & $u_{C 00}$ & average arm capacitor voltage \\
\hline$p_{\alpha 0}, p_{\beta 0}$ & vertical arm power & $\Rightarrow$ & $u_{C \alpha 0}, u_{C \beta 0}$ & vertical arm capacitor voltage \\
\hline$p_{0 \alpha}, p_{0 \beta}$ & horizontal arm power & $\Rightarrow$ & $u_{C 0 \alpha}, u_{C 0 \beta}$ & horizontal arm capacitor voltage \\
\hline$p_{d 1 \alpha}, p_{d 1 \beta}$ & diagonal 1 arm power & $\Rightarrow$ & $u_{C d 1 \alpha}, u_{C d 1 \beta}$ & diagonal 1 arm capacitor voltage \\
\hline$p_{d 2 \alpha}, p_{d 2 \beta}$ & diagonal 2 arm power & $\Rightarrow$ & $u_{C d 2 \alpha}, u_{C d 2 \beta}$ & diagonal 2 arm capacitor voltage \\
\hline
\end{tabular}

After replacing the arm voltages $u_{x y}$ and currents $i_{x y}$ by their transformed components, the voltage and current pairs for the power generation in each direction can be identified. To simplify the following analysis, sinusoidal voltages and currents are assumed. The Cartesian components of the input currents $i_{e \alpha}, i_{e \beta}$ and voltages $u_{L \alpha}, u_{L \beta}$ are expressed by their polar coordinates:

$$
\begin{array}{ll}
u_{L \alpha}=\hat{U}_{L} \cdot \cos \left(\gamma_{e}\right) & i_{e \alpha}=\hat{I}_{e} \cdot \cos \left(\gamma_{e}+\varphi_{e}\right) \\
u_{L \beta}=\hat{U}_{L} \cdot \sin \left(\gamma_{e}\right) & i_{e \beta}=\hat{I}_{e} \cdot \sin \left(\gamma_{e}+\varphi_{e}\right)
\end{array}
$$

$\hat{U}_{L}$ and $\hat{I}_{e}$ are the amplitudes of the input voltages and currents, $\gamma_{e}$ is the phase angle of the three-phase voltage and $\varphi_{e}$ is the phase shift between the input voltage and current. The output currents $i_{a \alpha}, i_{a \beta}$ and voltages $u_{a \alpha}, u_{a \beta}$ can be expressed in the same manner by their polar coordinates:

$$
\begin{array}{ll}
u_{a \alpha}=\hat{U}_{a} \cdot \cos \left(\gamma_{a}+\gamma_{a 0}\right) & i_{a \alpha}=\hat{I}_{a} \cdot \cos \left(\gamma_{a}+\gamma_{a 0}+\varphi_{a}\right) \\
u_{a \beta}=\hat{U}_{a} \cdot \sin \left(\gamma_{a}+\gamma_{a 0}\right) & i_{a \beta}=\hat{I}_{a} \cdot \sin \left(\gamma_{a}+\gamma_{a 0}+\varphi_{a}\right)
\end{array}
$$

$\hat{U}_{a}$ and $\hat{I}_{a}$ are the amplitudes of the output voltages and currents, $\gamma_{a}$ is the phase angle of the three-phase voltage with a initial phase shift $\gamma_{a 0}$ between the input and output voltage system and $\varphi_{a}$ is the phase shift between the output voltage and current. The internal diagonal currents $i_{d 1 \alpha}, i_{d 1 \beta}, i_{d 2 \alpha}, i_{d 2 \beta}$ do not influence the input or the output side of the M3C. They are degrees of freedom and can be expressed by their polar coordinates:

$$
\begin{array}{ll}
i_{d 1 \alpha}=\hat{I}_{d 1} \cdot \cos \left(\gamma_{d 1}\right) & i_{d 2 \alpha}=\hat{I}_{d 2} \cdot \cos \left(\gamma_{d 2}\right) \\
i_{d 1 \beta}=\hat{I}_{d 1} \cdot \sin \left(\gamma_{d 1}\right) & i_{d 2 \beta}=\hat{I}_{d 2} \cdot \sin \left(\gamma_{d 2}\right)
\end{array}
$$


Table II: Transformed arm power component generation overview with the resulting phase angles. Symbols: $\Sigma=$ sum angle component, $\Delta=$ difference angle component, $\circlearrowleft=$ positive sequence, $\circlearrowright=$ negative sequence

\begin{tabular}{|c|c|c|c|c|c|c|c|}
\hline line & $u$ & $i$ & $p_{00}$ & $p_{\alpha 0}, p_{\beta 0}$ & $p_{0 \alpha}, p_{0 \beta}$ & $p_{d 1 \alpha}, p_{d 1 \beta}$ & $p_{d 2 \alpha}, p_{d 2 \beta}$ \\
\hline 1 & $\begin{array}{c}\hat{U}_{L} \circlearrowleft \\
\gamma_{e}\end{array}$ & $\begin{array}{l}\hat{I}_{e} / 3 \circlearrowleft \\
\gamma_{e}+\varphi_{e}\end{array}$ & $\begin{array}{l}\Delta \\
\varphi_{e}\end{array}$ & $\begin{array}{c}\Sigma \circlearrowright \\
2 \gamma_{e}+\varphi_{e}\end{array}$ & & & \\
\hline 2 & $\begin{array}{c}\hat{U}_{a} \circlearrowleft \\
\gamma_{a}\end{array}$ & $\begin{array}{l}\hat{I}_{e} / 3 \circlearrowleft \\
\gamma_{e}+\varphi_{e}\end{array}$ & & & & $\begin{array}{c}\Delta \circlearrowleft \\
\gamma_{a}-\gamma_{e}-\varphi_{e}\end{array}$ & $\begin{array}{c}\Sigma \circlearrowleft \\
\gamma_{a}+\gamma_{e}+\varphi_{e}\end{array}$ \\
\hline 3 & $\begin{array}{c}\hat{U}_{L} \circlearrowleft \\
\gamma_{e} \\
\end{array}$ & $\begin{array}{l}\hat{I_{a} / 3 \circlearrowleft} \\
\gamma_{a}+\varphi_{a}\end{array}$ & & & & $\begin{array}{c}\Delta \circlearrowleft \\
\gamma_{a}+\varphi_{a}-\gamma_{e}\end{array}$ & $\begin{array}{c}\Sigma \circlearrowleft \\
\gamma_{a}+\varphi_{a}+\gamma_{e}\end{array}$ \\
\hline 4 & $\begin{array}{l}\hat{U}_{a} \circlearrowleft \\
\gamma_{a} \\
\end{array}$ & $\begin{array}{l}\hat{I}_{a} / 3 \circlearrowleft \\
\gamma_{a}+\varphi_{a} \\
\end{array}$ & $\begin{array}{l}\Delta \\
\varphi_{a} \\
\end{array}$ & & $\begin{array}{c}\Sigma \circlearrowright \\
2 \gamma_{a}+\varphi_{a} \\
\end{array}$ & & \\
\hline 5 & $\begin{array}{c}\hat{U}_{L} \circlearrowleft \\
\gamma_{e}\end{array}$ & $\begin{array}{c}\hat{I}_{d 1} \circlearrowleft \\
\gamma_{d 1}\end{array}$ & & & $\begin{array}{c}\sum \circlearrowleft \\
\gamma_{e}+\gamma_{d 1}\end{array}$ & & $\begin{array}{c}\Delta \circlearrowleft \\
\gamma_{d 1}-\gamma_{e}\end{array}$ \\
\hline 6 & $\begin{array}{c}\hat{U_{a} \circlearrowleft} \\
\gamma_{a} \\
\end{array}$ & $\begin{array}{c}\hat{I}_{d 1} \circlearrowleft \\
\gamma_{d 1} \\
\end{array}$ & & $\begin{array}{c}\Delta \circlearrowright \\
\gamma_{d 1}-\gamma_{a} \\
\end{array}$ & & & $\begin{array}{c}\Sigma \circlearrowright \\
\gamma_{a}+\gamma_{d 1} \\
\end{array}$ \\
\hline 7 & $\begin{array}{c}\hat{U}_{L} \circlearrowleft \\
\gamma_{e}\end{array}$ & $\begin{array}{l}\hat{I}_{d 2} \circlearrowleft \\
\gamma_{d 2}\end{array}$ & & & $\begin{array}{c}\Delta \circlearrowleft \\
\gamma_{d 2}-\gamma_{e}\end{array}$ & $\begin{array}{c}\Sigma \circlearrowleft \\
\gamma_{e}+\gamma_{d 2}\end{array}$ & \\
\hline 8 & $\begin{array}{l}\hat{U_{a} \circlearrowleft} \\
\gamma_{a} \\
\end{array}$ & $\begin{array}{l}\hat{I}_{d 2} \circlearrowleft \\
\gamma_{d 2} \\
\end{array}$ & & $\begin{array}{c}\Delta \circlearrowleft \\
\gamma_{d 2}-\gamma_{a} \\
\end{array}$ & & $\begin{array}{c}\Sigma \circlearrowright \\
\gamma_{a}+\gamma_{d 2} \\
\end{array}$ & \\
\hline 9 & $\begin{array}{l}\hat{U}_{0} \\
\gamma_{u 0}\end{array}$ & $\begin{array}{l}\hat{I}_{e} / 3 \circlearrowleft \\
\gamma_{e}+\varphi_{e}\end{array}$ & & $\begin{array}{c}\Sigma \circlearrowleft, \Delta \circlearrowright \\
\gamma_{u 0} \pm\left(\gamma_{e}+\varphi_{e}\right)\end{array}$ & & & \\
\hline 10 & $\begin{array}{l}\hat{U}_{0} \\
\gamma_{u 0} \\
\end{array}$ & $\begin{array}{l}\hat{I}_{a} / 3 \circlearrowleft \\
\gamma_{a}+\varphi_{a} \\
\end{array}$ & & & $\begin{array}{c}\sum \circlearrowleft, \Delta \circlearrowright \\
\gamma_{u 0} \pm\left(\gamma_{a}+\varphi_{a}\right)\end{array}$ & & \\
\hline 11 & $\begin{array}{l}\hat{U}_{0} \\
\gamma_{u 0}\end{array}$ & $\begin{array}{c}\hat{I}_{d 1} \circlearrowleft \\
\gamma_{d 1}\end{array}$ & & & & $\begin{array}{l}\Sigma \circlearrowleft, \Delta \circlearrowright \\
\gamma_{u 0} \pm \gamma_{d 1}\end{array}$ & \\
\hline 12 & $\begin{array}{l}\hat{U}_{0} \\
\gamma_{u 0} \\
\end{array}$ & $\begin{array}{c}\hat{I}_{d 2} \circlearrowleft \\
\gamma_{d 2} \\
\end{array}$ & & & & & $\begin{array}{l}\Sigma \circlearrowleft, \Delta \circlearrowright \\
\gamma_{u 0} \pm \gamma_{d 2} \\
\end{array}$ \\
\hline
\end{tabular}

$\hat{I}_{d 1}, \hat{I}_{d 2}$ represent the amplitudes of the diagonal 1 and 2 current systems. $\gamma_{d 1}, \gamma_{d 2}$ are the phase angles of the diagonal 1 and 2 current systems. Note that the diagonal 1 and 2 current systems can be superposed by several independent systems with different amplitudes $\hat{I}_{d 1}, \hat{I}_{d 2}$ and phase angles $\gamma_{d 1}$ and $\gamma_{d 2}$.

The zero sequence voltage $u_{0}$ is an additional degree of freedom:

$$
u_{0}=\hat{U}_{0} \cdot \cos \left(\gamma_{u 0}\right)
$$

$\hat{U}_{0}$ is the amplitude of the zero sequence voltage and $\gamma_{u 0}$ the phase angle. Again several superposed zero sequence voltage systems can be used if necessary.

Applying the voltage and currents definitions from (23) - (26) to the transformed arm power components from Table I results in the power components shown in Table II. Table II is organized as follows:

- The colors of the voltage, current and power components corresponds to the four directions and the average value of Fig. 1.

- Each line contains in column 2 the corresponding voltage amplitude and phase angle according to (23), (24), (26). The rotational direction are defined as positive sequence and symbolized with $\circlearrowleft$.

- Each line contains in column 3 the corresponding current amplitude and phase angle according to (23) - (25). The rotational direction are defined as positive sequence and symbolized with $\circlearrowleft$.

- Column 4 contain the average power components of Fig. 1. Line 1 corresponds to the arm input power generated by the input voltage and current with the phase shift $\varphi_{e}$. Line 4 corresponds to the arm output power generated by the output voltage and current with the phase shift $\varphi_{a}$.

- The Columns $5-8$ contain the resulting power components for the four directions of Fig. 1. They can be interpreted as power space vectors with an amplitude and phase angle. Here each voltage current pair generates two power components located at different directions and containing the sum and the difference of the phase angles from the voltage and current.

- The power component with the sum angle is marked with $\Sigma$ and the power component with the difference angle is marked with $\Delta$. The rotational directions can be a positive sequence symbolized with $\circlearrowleft$ or a negative sequence symbolized with $\circlearrowright$. The amplitudes of the power components can be calculated for each line by multiplying the corresponding voltage and current amplitudes: $\hat{P}=\frac{1}{2} \hat{U} \hat{I}$. 
The following observations can be made from Table II:

A minimum of eight power components occurs by applying only input $i_{e}$ and output $i_{a}$ currents (line 1 - 4). With $\gamma_{e}=\omega_{e} \cdot t\left(\omega_{e}\right.$ : input angular frequency, $t$ : time) and $\gamma_{a}=\omega_{a} \cdot t+\gamma_{a 0}\left(\omega_{a}\right.$ : output angular frequency, $\gamma_{a 0}$ : initial phase angle between the input and the output voltage), the active input and output power components influences $p_{00}$ and thereby $u_{C 00}$ (column 4). To maintain a constant average arm capacitor voltage $\bar{u}_{C x y}$, all $p_{00}$ components must sum up to zero. The six reactive power components are separated into the angular frequencies $2 \omega_{e}, 2 \omega_{a}, \omega_{a}-\omega_{e}$ and $\omega_{a}+\omega_{e}$. Each of them is located to one of the four directions. They must be buffered in the cell capacitors $C_{x y z}$ and cause voltage pulsations in their respective directions. This pulsations can be calculated in real time by using the primitives of the reactive power terms. With this information, a separation of the DC components and the AC components can be done very fast and without any filters in the feedback paths of the balancing controllers. During a stationary operation of the $\mathrm{M} 3 \mathrm{C}$ with $\left|\omega_{a}\right| \neq\left|\omega_{e}\right|$, no active power component occur in the four directions, hence no balancing is necessary. For an operation at the same angular frequency $\omega_{a}=\omega_{e}$, the reactive power components $p_{d 1 \alpha}, p_{d 1 \beta}$ in diagonal 1 direction (line 2 and 3 ) changes to active power components which must be compensated to zero to ensure a symmetrical operation. The same occurs for a operation at the same absolute angular frequency $\omega_{a}=-\omega_{e}$ with the power components $p_{d 2 \alpha}, p_{d 2 \beta}$ in diagonal 2 direction (line 2 and 3). The solution of this problem is shown in Section 3.

The internal diagonal 1 and 2 currents $i_{d 1}, i_{d 2}$ can be used to generate eight power components with the input voltage $u_{L}$ and the output voltage $u_{a}$ (line $5-8$ ). By a proper selection of the current phase angles $\gamma_{d 1}$ and $\gamma_{d 2}$ and amplitudes $\hat{I}_{d 1}, \hat{I}_{d 2}$, active power components for the balancing control and reactive power components for a reduction of the energy pulsation can be generated in each of the four directions.

Additionally, the zero sequence voltage $u_{0}$ with the phase angle $\gamma_{u 0}$ can be used to generate power components in all four balancing directions (line $9-12$ ). The zero sequence voltage $u_{0}$ generates power components with all four currents, for this reason it should be used only if necessary to avoid additional reactive power components which must be buffered by the cell capacitors.

\section{Operation with equal input and output frequency}

For the M3C operation at the same absolute angular frequency $\left|\omega_{a}\right|=\omega_{e}$, the reactive power components from line 2 and 3 Table II in diagonal 1 or diagonal 2 direction change to active power components. Hence they must be compensated to allow a stable operation of the M3C. As an attempt to solve this problem Kawamura et al. presented a solution for diagonal 1 direction using an adjustment of the input current phase angle $\varphi_{e}=-\varphi_{a}$ by applying reactive power at the input side for $\omega_{a}=\omega_{e}$ [12]. Experimental results shows a successful $50 \mathrm{~Hz}-48 \mathrm{~Hz}$ conversion and compensation of the reactive power with $\omega_{e}-$ $\omega_{a}=2 \mathrm{~Hz}$. In this paper, the solution is extended for an operation at the same absolute angular frequency $\omega_{a}=-\omega_{e}$. Additionally, an alternative balancing method using the zero sequence voltage $u_{0}$ is presented. Table III shows the possibilities to compensate the diagonal 1 power components $p_{d 1 \alpha}, p_{d 1 \beta}$ for $\omega_{a} \approx \omega_{e}$. Line 2 and 3 contain the power components which must be compensated. This can be done by using two superposed internal diagonal currents $i_{d 2,1}$ and $i_{d 2,2}$ with the phase angles shown in line 7 and 8 . Additionally, it is necessary to adjust the input phase angle $\varphi_{e}=-\varphi_{a}$ by applying reactive power at the input side to achieve a full compensation [12]. Alternatively line 11 can be used in cases there the reactive power at the input side is not allowed. Here a zero sequence voltage $u_{0}$ with phase angle

Table III: Compensation of the power components for $\omega_{a} \approx \omega_{e}$, line 7 and 8: Solution presented by Kawamura et al. using $i_{d 2}$ [12], line 11: Solution presented in this paper using $i_{d 1}$ and $u_{0}$.

\begin{tabular}{|c|c|c|c|c|}
\hline line Table II & $u$ & $i$ & $p_{d 1 \alpha}, p_{d 1 \beta}(\mathrm{d} 1)$ & Comment \\
\hline \hline 2 & $\hat{U}_{a} \circlearrowleft$ & $\hat{I}_{e} / 3 \circlearrowleft$ & $\Delta \circlearrowleft$ & unwanted power \\
& $\gamma_{a}$ & $\gamma_{e}+\varphi_{e}$ & $\gamma_{P d 1,1}=\gamma_{a}-\gamma_{e}-\varphi_{e}$ & component \\
\hline \hline 3 & $\hat{U}_{L} \circlearrowleft$ & $\hat{I}_{a} / 3 \circlearrowleft$ & $\Delta \circlearrowleft$ & unwanted power \\
& $\gamma_{e}$ & $\gamma_{a}+\varphi_{a}$ & $\gamma_{P d 1,2}=\gamma_{a}+\varphi_{a}-\gamma_{e}$ & compoment \\
\hline \hline 7 & $\hat{U}_{L} \circlearrowleft$ & $\hat{I}_{d 2,2} \circlearrowleft$ & $\Sigma \circlearrowleft$ & Compensates line 3 \\
& $\gamma_{e}$ & $-\gamma_{e}+\gamma_{P d 1,2}$ & $\gamma_{P d 1,2}=\gamma_{a}+\varphi_{a}-\gamma_{e}$ & $\varphi_{e}=-\varphi_{a}$ required [12] \\
\hline \hline \multirow{2}{*}{11} & $\hat{U}_{a} \circlearrowleft$ & $\hat{I}_{d 2,1} \circlearrowleft$ & $\sum \circlearrowleft$ & Compensates line 2 \\
& $\gamma_{a}$ & $-\gamma_{a}-\gamma_{P d 1,1}$ & $\gamma_{P d 1,1}=\gamma_{a}-\gamma_{e}-\varphi_{e}$ & $\varphi_{e}=-\varphi_{a}$ required [12] \\
\hline \multirow{2}{*}{11} & $\hat{U}_{0}$ & $\hat{I}_{d 1,1} \circlearrowleft$ & $\sum \circlearrowleft, \Delta \circlearrowright$ & Compensates line 2 \\
& $\gamma_{u 0}$ & $\pm \gamma_{u 0}+\gamma_{P d 1,1}$ & $\gamma_{P d 1,1}=\gamma_{a}-\gamma_{e}-\varphi_{e}$ & \\
& $\hat{U}_{0}$ & $\hat{I}_{d 1,2} \circlearrowleft$ & $\Sigma \circlearrowleft, \Delta \circlearrowright$ & Compensates line 3 \\
& $\gamma_{u 0}$ & $\pm \gamma_{u 0}+\gamma_{P d 1,2}$ & $\gamma_{P d 1,2}=\gamma_{a}+\varphi_{a}-\gamma_{e}$ & \\
\hline
\end{tabular}


$\gamma_{u 0}=\omega_{u 0} \cdot t \neq \omega_{e} \cdot t\left(\omega_{u 0}\right.$ : zero sequence voltage angular frequency) is applied. Then two independent internal diagonal currents $i_{d 1,1}$ and $i_{d 1,2}$ are modulated according to line 11 to achieve the compensation without any reactive power.

Applying the same rules for $\omega_{a} \approx-\omega_{e}$ results in Table IV. Here only the diagonal currents are changed, but the compensation works similar to Table III. Internal diagonal currents $i_{d 1,1}$ and $i_{d 1,2}$ can be used with the phase angles shown in line 5 and 6 . Additionally, it is necessary to adjust the input phase angle $\varphi_{e}=\varphi_{a}$ by applying reactive power at the input side to achieve a full compensation. This is an extension of the method presented in[12] for negative output angular frequencies. Line 12 presents the solution with a zero sequence voltage $u_{0}$ together with two superposed internal diagonal currents $\hat{I}_{d 2,1}$ and $\hat{I}_{d 2,2}$.

Table IV: Compensation of the power components for $\omega_{a} \approx-\omega_{e}$, line 5 and 6: Solution using $i_{d 1}$, line 12: Solution presented in this paper using $i_{d 2}$ and $u_{0}$.

\begin{tabular}{|c|c|c|c|c|}
\hline line Table II & $u$ & $i$ & $p_{d 2 \alpha}, p_{d 2 \beta}(\mathrm{d} 2)$ & Comment \\
\hline \hline 2 & $\hat{U}_{a} \circlearrowleft$ & $\hat{I}_{e} / 3 \circlearrowleft$ & $\Sigma \circlearrowleft$ & unwanted power \\
& $\gamma_{a}$ & $\gamma_{e}+\varphi_{e}$ & $\gamma_{P d 2,1}=\gamma_{a}+\gamma_{e}+\varphi_{e}$ & component \\
\hline \hline 3 & $\hat{U}_{L} \circlearrowleft$ & $\hat{I}_{a} / 3 \circlearrowleft$ & $\Sigma \circlearrowleft$ & unwanted power \\
& $\gamma_{e}$ & $\gamma_{a}+\varphi_{a}$ & $\gamma_{P d 2,2}=\gamma_{a}+\varphi_{a}+\gamma_{e}$ & component \\
\hline \hline 5 & $\hat{U}_{L} \circlearrowleft$ & $\hat{I}_{d 1,2} \circlearrowleft$ & $\Delta \circlearrowleft$ & Compensates line 3 \\
& $\gamma_{e}$ & $\gamma_{e}+\gamma_{P d 2,2}$ & $\gamma_{P d 2,2}=\gamma_{a}+\varphi_{a}+\gamma_{e}$ & $\varphi_{e}=\varphi_{a}$ required \\
\hline \multirow{2}{*}{6} & $\hat{U}_{a} \circlearrowleft$ & $\hat{I}_{d 1,1} \circlearrowleft$ & $\Sigma \circlearrowright$ & Compensates line 2 \\
& $\gamma_{a}$ & $-\gamma_{a}-\gamma_{P d 2,1}$ & $\gamma_{P d 2,1}=\gamma_{a}+\gamma_{e}+\varphi_{e}$ & $\varphi_{e}=\varphi_{a}$ required \\
\hline \hline \multirow{2}{*}{12} & $\hat{U}_{0}$ & $\hat{I}_{d 2,1} \circlearrowleft$ & $\Sigma \circlearrowleft, \Delta \circlearrowright$ & Compensates line 2 \\
& $\gamma_{u 0}$ & $\pm \gamma_{u 0}+\gamma_{P d 2,1}$ & $\gamma_{P d 2,1}=\gamma_{a}+\gamma_{e}+\varphi_{e}$ & \\
& $\hat{U}_{0}$ & $\hat{I}_{d 2,2} \circlearrowleft$ & $\Sigma \circlearrowleft, \Delta \circlearrowright$ & Compensates line 3 \\
& $\gamma_{u 0}$ & $\pm \gamma_{u 0}+\gamma_{P d 2,2}$ & $\gamma_{P d 2,2}=\gamma_{a}+\varphi_{a}+\gamma_{e}$ & \\
\hline
\end{tabular}

With the solution of this paper, a $\left|\omega_{a}\right| \approx \omega_{e}$ operation of the M3C is possible under all (!) circumstances. The additional voltage margin for the zero sequence voltage $u_{0}$ and the additional currents stress for the internal currents must be considered in the dimensioning. Otherwise the output power capability is limited in this operation points.

\section{Experimental results}

To demonstrate the performance of the M3C, a low voltage laboratory prototype was built up with $N=5$ cells per arm. The control method from [8] was extended with the feed forward calculation of the compensation currents from Table III and IV. The balancing current or voltage for each of the four directions was selected based on Table II under consideration of the unwanted coupling effect which occurs for $\left|\omega_{a}\right| \approx \omega_{e}$. Here line 11 and 12 are used for the diagonal balancing control using a zero sequence voltage of $\hat{U}_{0}=100 \mathrm{~V}$ with an angular frequency of $\omega_{0}=100 \mathrm{~Hz}$. Fig. 3 shows the measurement results for a $50 \mathrm{~Hz} / 50 \mathrm{~Hz}$ conversion. All nine arm capacitor voltages $u_{C x y}$ are balanced and the average value $u_{C 00}=600 \mathrm{~V}$ is constant. Hence the average values of the transformed arm capacitor voltages in the four directions are zero. The input voltage amplitude is $\hat{U}_{L}=285 \mathrm{~V}$ and the output voltage amplitude is $\hat{U}_{a}=200 \mathrm{~V}$ and the power $P_{a}=4 \mathrm{~kW}$ is transferred to a resistive load. Therefore a successful and stable operation of the $\mathrm{M} 3 \mathrm{C}$ is shown for the most critical operation point.

\section{Conclusion}

In this paper, the transformed arm power analysis of the Modular Multilevel Matrix Converter (M3C) is introduced. It allows an easier design of the current control and the energy balancing of all nine converter arms. Furthermore, the necessary internal currents and zero sequence voltage for the operation with equal input and output frequency are calculated. Measurements on a low voltage laboratory prototype show the control performance for this operation point. With the method of this paper, the M3C is able to work in the whole frequency range for grid or motor drive applications. The operating range includes equal frequencies on the input and the output side as well as any combinations of positive and negative sequence three phase systems. 


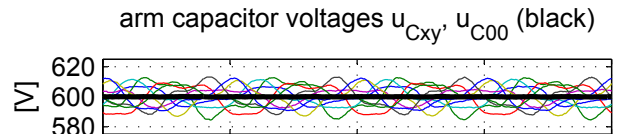

vertical arm capacitor voltages $\mathrm{u}_{\mathrm{C} \alpha 0}, \mathrm{u}_{\mathrm{C} \beta 0}$

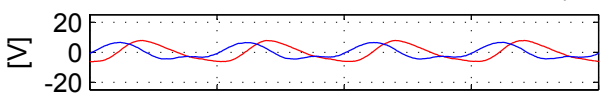

vertical arm capacitor voltages $\mathrm{u}_{\mathrm{CO} \alpha}, \mathrm{u}_{\mathrm{C} 0 \beta}$

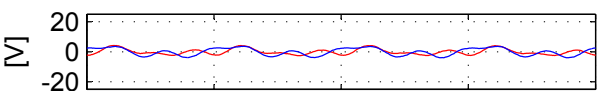

diagonal 1 arm capacitor voltages $\mathrm{u}_{\mathrm{Cd} 1 \alpha}, \mathrm{u}_{\mathrm{Cd} 1}$

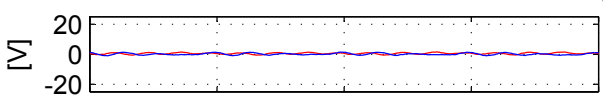

$-20$
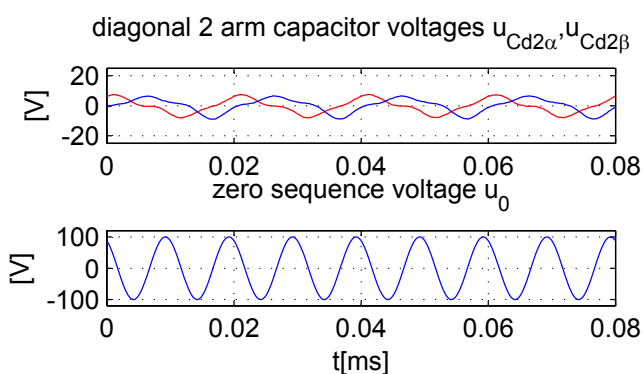

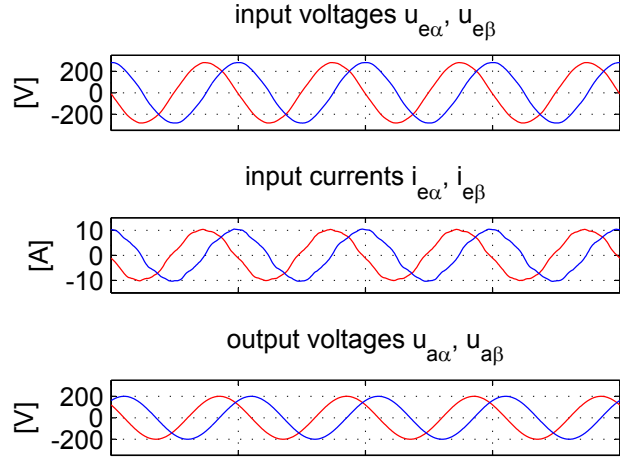

output currents $\mathrm{i}_{\mathrm{a \alpha}}, \mathrm{i}_{\mathrm{a} \beta}$

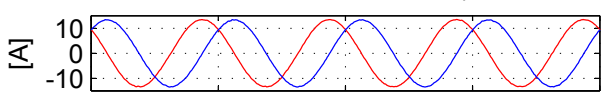

diagonal 1 currents $\mathrm{i}_{\mathrm{d} 1 \alpha}, \mathrm{i}_{\mathrm{d} 1 \beta}$

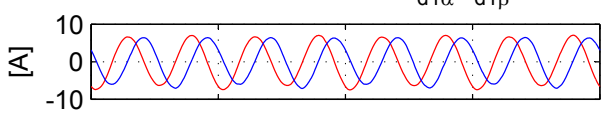

diagonal 2 currents $i_{d 2 \alpha}, i_{d 2 \beta}$

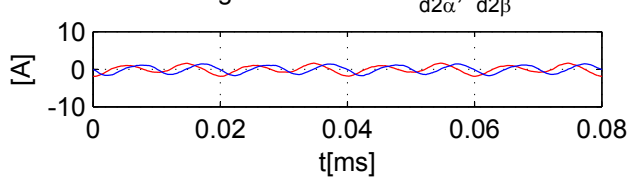

Figure 3: Measurement of the $\mathrm{M} 3 \mathrm{C}$ control variables doing the $50 \mathrm{~Hz}$ to $50 \mathrm{~Hz}$ conversion, short time averaged values at $t_{A}=\frac{1}{f_{P W M}}=\frac{1}{8 K H z}$.

\section{References}

[1] S. Angkititrakul and R.W. Erickson.: Control and implementation of a new modular matrix converter, In Applied Power Electronics Conference and Exposition, 2004. APEC 04.

[2] Miura, Y., Mizutani, T., Ito, M. and Ise, T.: Modular multilevel matrix converter for low frequency AC transmission, Power Electronics and Drive Systems (PEDS), 2013 IEEE 10th International Conference on.

[3] Oates, C.: A methodology for developing Chainlink converters, Power Electronics and Applications, 2009. EPE '09. 13th European Conference on.

[4] Oates, C. and Mondal, G.: DC circulating Current for Capacitor Voltage Balancing in Modular Multilevel Matrix Converter, EPE 2011 Birmingham.

[5] Ludois, D. C., Reed, J. K. and Venkataramanan, G.: Hierarchical Control of Bridge-of-Bridge Multilevel Power Converters, Industrial Electronics, IEEE Transactions on, 57(8):2679 - 2690, aug. 2010

[6] Korn, A.J., Winkelnkemper, M., Steimer, P. and Kolar, J.W.: Direct modular multi-level converter for gearless low-speed drives, Power Electronics and Applications (EPE 2011), Proceedings of the 2011-14th European Conference on.

[7] Kammerer, F., Kolb, J. and Braun, M.: A novel cascaded vector control scheme for the Modular Multilevel Matrix Converter, IECON 2011 Melbourne.

[8] Kammerer, F., Kolb, J. and Braun, M.: Fully decoupled current control and energy balancing of the Modular Multilevel Matrix Converter, EPE-PEMC 2012 ECCE Europe, Novi Sad, Serbia.

[9] Kammerer, F., Kolb, J. and Braun, M.: Optimization of the passive components of the Modular Multilevel Matrix Converter for Drive Applications, PCIM Europe 2012, Nuremberg, Germany.

[10] Kammerer, F., Gommeringer, M., Kolb, J. and Braun, M.: Benefits of Operating Doubly Fed Induction Generators by Modular Multilevel Matrix Converters, PCIM Europe 2013, Nuremberg, Germany.

[11] Kawamura, W., Hagiwara, M. and Akagi, H.: Control and Experiment of a Modular Multilevel Cascade Converter Based on Triple-Star Bridge Cells (MMCC-TSBC), Industry Applications, IEEE Transactions on, PP(99):1-1,2014.

[12] Kawamura, W., Hagiwara, M. and Akagi, H.: A broad range of frequency control for the modular multilevel cascade converter based on triple-star bridge-cells (MMCC-TSBC), Energy Conversion Congress and Exposition (ECCE), 2013 IEEE.

[13] Kawamura, W., Hagiwara, M. and Akagi, H.: Experimental verification of a modular multilevel cascade converter based on triple-star bridge-cells (MMCC-TSBC) for motor drives, Future Energy Electronics Conference (IFEEC), 2013 1st International. 